\title{
Research on Fourth Harmonic Mixer at W Band in the Imaging System
}

\author{
Bo Xiang $\cdot$ Wenbin Dou $\cdot$ Minmin He $\cdot$ Zongxin Wang
}

\begin{abstract}
This paper presents a novel fourth harmonic mixer with new structure. The traditional 3-ports fourth harmonic mixer and the novel fourth harmonic mixer are designed by ADS, HFSS and CST simulator. The mixers have been fabricated and tested. The size of the traditional 3-ports fourth harmonic mixer is $12 \times 15 \mathrm{~mm}$, and the best conversion loss is $18.7 \mathrm{~dB}$ according to the measurement. Since the traditional 3-port mixer size is too large to be ranked, we design a novel fourth harmonic mixer for imaging system. The width of the mixing module in the novel fourth harmonic mixer is only $3.65 \mathrm{~mm}$, and this size is fully capable to meet the mixer unit space which is not greater than $5 \mathrm{~mm}$. The simulation result shows that the mixer has good performance, and the experiment result shows that the best conversion loss of the novel fourth harmonic mixer is $16.3 \mathrm{~dB}$ at RF signal of $91.3 \mathrm{GHz}$.
\end{abstract}

Key words: $3 \mathrm{~mm}$ Wave Length, Harmonic Mixer, Conversion Loss, Anti-Parallel Schottky Diode.

\section{Introduction}

The frequency of millimeter wave is between infrared wave and microwave. The millimeter-wave radiation detection equipment is more effective than photoelectric detector especially in inclement weather or dusty conditions [1, 2]. Millimeter-wave radiation detection technology is playing an important role in many fields and can work in all-weather. Direct detection receiver is used in many millimeter-wave imaging systems. Although direct detection receiver does not require local oscillators and has the advantages of low DC power consumption and simple structure, it can only extract amplitude information. The holographic imaging system is used to obtain better imaging quality. As one of the key components in the holographic millimeter-wave imaging system, the mixer performance is very important. In order to improve imaging speed, we designed an array composing of multiple mixers to receive and process image information.

In 1975, harmonic mixer was reported in [3] firstly. In 2008, the fourth harmonic mixer at Ka band was proposed and the minimum loss is 7.4 [4]. In 2009, a Wband eighth-order harmonic mixer achieved a conversion loss of $36 \pm 6 \mathrm{~dB}$ across a frequency range of $80 \sim$ $105 \mathrm{GHz}$ [5]. A $75 \sim 110 \mathrm{GHz}$ seventh-harmonic balanced diode mixer had very flat characteristics of conversion loss of about $19 \pm 2.5 \mathrm{~dB}$ over the entire W- band [6]. Two kinds of fourth harmonic mixer are present in this paper. According to the experiment results, the minimum conversion loss of the novel fourth harmonic mixer is only $16.3 \mathrm{~dB}$. Moreover, the size of the novel fourth harmonic mixer is smaller than the former mixers. The most specific character of novel fourth harmonic mixer is that it can be arrayed to be implemented in the imaging system.

Firstly, we fabricated and tested a traditional 3-ports fourth harmonic mixer to demonstrate the feasibility of holographic imaging. Secondly, in imaging systems we need place many mixers on the focal plane to receive RF signals, as shown in Fig. 6. According to Nyquist theorem, the mixing unit space should be less than 1.5 $\mathrm{mm}$, the traditional 3-ports mixer is hard to meet the requirement. Considering manufacturing technique, we assume that the mixers unit space of design specifications is $5 \mathrm{~mm}$, and designed the novel fourth harmonic mixer with new structure. In this paper, the novel harmonic mixer is divided into two modules, as shown in Fig. 7. One module is 2-ports component containing a GaAs flip chip anti-parallel pair Schottky diode, the other is a diplexer.

\section{The Traditional 3-Ports Fourth Sub-Harmonic Mixer}

\section{2-1 Design and Simulation}

The traditional fourth harmonic mixer, as shown in Fig. 1 [4 7], consists of four open/short stubs at the two sides of the anti-parallel pair Schottky diode. RF signal is fed to left side of the diode and LO signal is fed to right side. The IF signals is taken out from the RF port. The waveguide/microstrip transition works as not only

Manuscript received April 14, 2010 ; revised November 12, 2010. (ID No. 20100414-15J)

State Key Laboratory of Millimeter Waves, Southeast University, Nanjing, China.

Corresponding Author : Bo Xiang (e-mail : njxiangbo@gmail.com) 


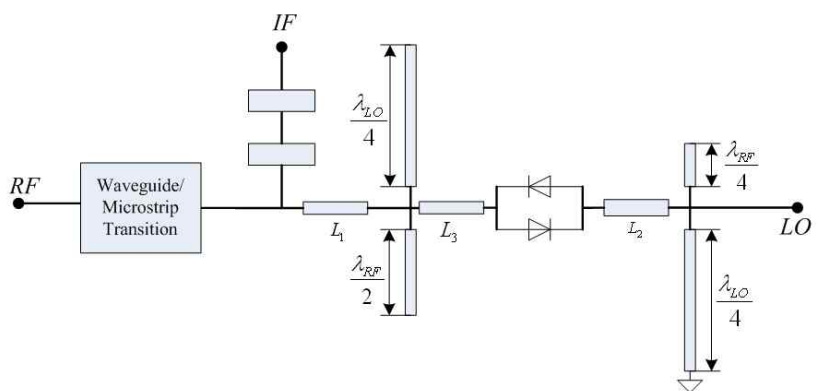

Fig. 1. The fourth sub-harmonic mixer diagram.

signal transition, but also bandpass filter to prevent the IF \& LO from leaking to RF. The lowpass filter is used to filter IF signal.

The waveguide/microstrip transition model [8] is built and simulated by HFSS in the Fig.2. Return loss of the RF signal between $88 \mathrm{GHz}$ and $101 \mathrm{GHz}$ is less than $-20 \mathrm{~dB}$ and insertion loss is very low. Reflection coefficient $S_{22}$ of the LO and IF signal between DC and $60 \mathrm{GHz}$ is approximately $0 \mathrm{~dB}$.

\section{2-2 Fabrication and Measurement}

The 3-port fourth harmonic mixer is shown in Fig. 3. The Schottky diode type is the MS8251-P261. The circuit is designed and fabricated on the RF-Duroid 5880 with thickness of $0.127 \mathrm{~mm}$ and dielectric constant of 2.22 .

The test diagram is the same as Fig. 11, and the de-
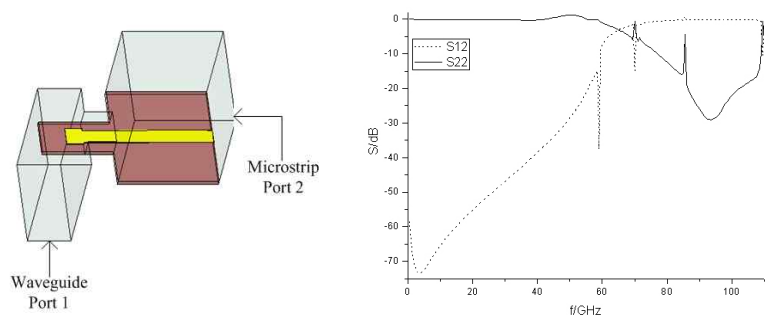

Fig. 2. Simulation model and simulation result of the waveguide/microstrip transition.


Fig. 3. Photograph of the 3-port sub-harmonic mixer.

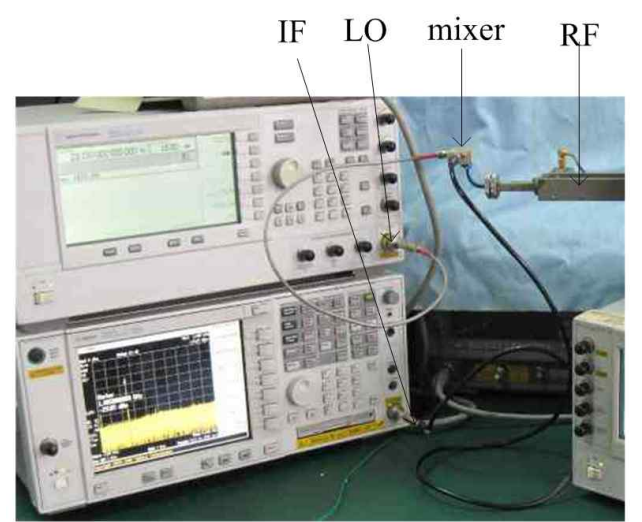

Fig. 4. Test scenario photograph of the 3-ports fourth harmonic mixer

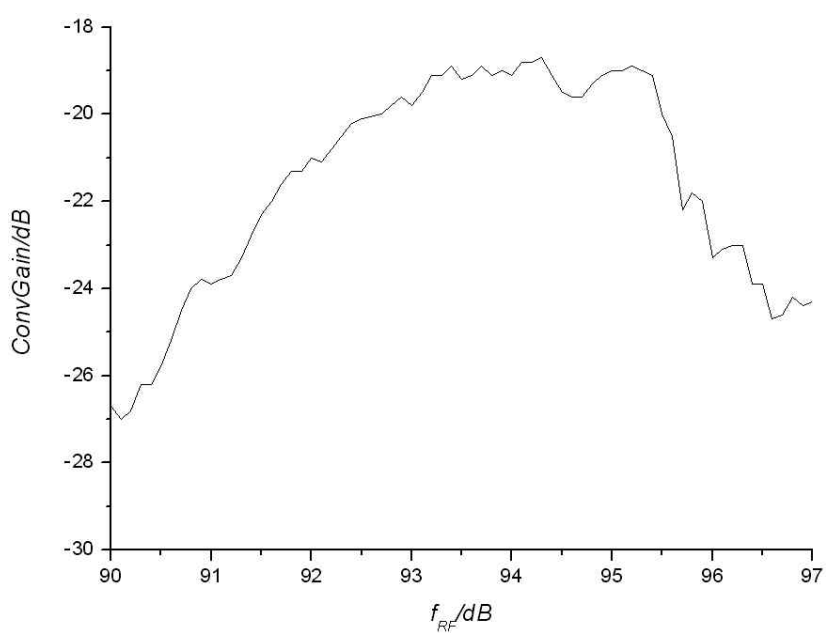

Fig. 5. Conversion loss measurement curve with the RF frequency.

tail description is in the Section III-4. The test scenario is shown in the Fig. 4.

The measurement result is shown in Fig. 5, when LO frequency is $23.15 \mathrm{GHz}$, the $\mathrm{LO}$ power is $14.2 \mathrm{dBm}$. When RF frequency is $94.3 \mathrm{GHz}$, the best conversion loss is $18.7 \mathrm{~dB}$. When RF frequency is between 92.7 $\mathrm{GHz}$ and $95.5 \mathrm{GHz}$, the conversion loss is less than 20 $\mathrm{dB}$, and the RF bandwidth is about $3 \mathrm{GHz}$.

\section{The Novel Fourth Harmonic Mixer}

\section{3-1 Application Background}

The millimeter wave imaging sketch is shown in Fig. 6. The electromagnetic waves radiating from imaged objects propagate through the lens and focus on the imaging plane. The mixer is ranked on the focus plane and the image is reconstructed by the mixer detecting information. We have tested traditional 3-ports fourth har- 


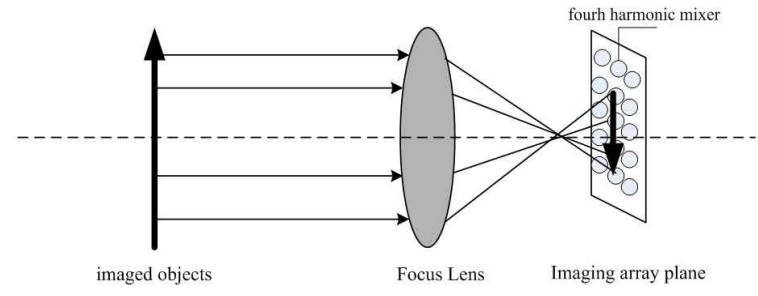

Fig. 6. Millimeter wave imaging sketch.

monic mixer which installed in a mechanical scanning equipment to get image. In order to improve scanning speed, the electronic scanning equipment including the fourth harmonic mixers array will have wide application. It is very difficult to design small size fourth harmonic mixer which can be ranked on the focus plane.

\section{3-2 Design}

The novel structure fourth harmonic mixer, as shown in Fig. 7, consists of anti-parallel pair diode mixer module and diplexer module. The design keys are as follows:

1) The output IF signal is delivered from the LO input port of the diode, which is different from the traditional fourth harmonic mixer.

2) The novel fourth harmonic mixer is divided into two modules, as shown in Fig. 7. One is the anti-parallel pair diode mixer module which is 2-port component. It is easy to place in a row of the focus plane. The other is the diplexer module which is used to isolate IF signal from LO signal. It is not required to be placed in a row.

3) One coaxial cable transmits LO and IF signals between the two modules mentioned above.

The basic circuit schematic has a $\lambda_{R F} / 4$ shortedcircuited stub on the RF side of the diode pair such that the stub provides a IF return path to ground, but the RF signal is not affected. Similarly, a $\lambda_{L O} / 4$ open circuited stub is located on the RF side of diode pair such that the diodes are terminated with a short circuit at the LO

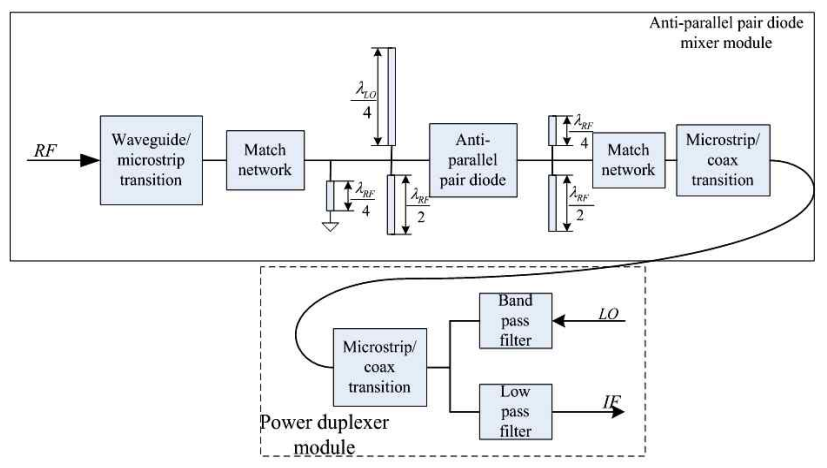

Fig. 7. Design diagram of the novel fourth harmonic mixer. frequency, but the RF signal is not affected. A $\lambda_{R F} / 4$ open-circuited stub on the LO side of the diode pair terminates diode at the RF frequency. The $\lambda_{R F} / 2$ opencircuited stubs on the both sides of the pair prevent signal of double LO frequency leakage to RF or LO, which enhance mixing efficiency.

The diplexer consists of a lowpass filter and a bandpass filter. The IF signal is extracted from the LO side of the diode pair. The $22.7 \sim 23.7 \mathrm{GHz}$ bandpass filter provide good rejection at $\mathrm{DC}-5 \mathrm{GHz}$, preventing IF leakage to the LO source.

\section{3-3 Simulation}

The anti-parallel pair diode mixer module is shown in Fig. 8. The maximum width is only $3.65 \mathrm{~mm}$, so it is easy to place in a row. The microstrip and stub are simulated and optimized by CST.

The diplexer module model is built and optimized by HFSS shown in Fig. 9. It consists of a lowpass filter and a bandpass filter. The lowpass filter is designed by using alternating section of very low and high characteristics impedance microstrip. The LO bandpass filter is designed by using microstrip series open-circuited stubs and its response is the same as the equivalent LC network [9].

The simulation results denoted by the hollow triangle are shown in Fig. 10. When the LO frequency is between $22.7 \mathrm{GHz}$ and $23.7 \mathrm{GHz}$, the reflection coefficient $S_{22}$ is less than $-20 \mathrm{~dB}$ and transmission coefficient $S_{12}$ is more than $-0.7 \mathrm{~dB}$. When the IF is between DC and $5 \mathrm{GHz}$, the transmission coefficient $S_{31}$ is more than $0.2 \mathrm{~dB}$. The isolation $S_{32}$ between LO input port and IF

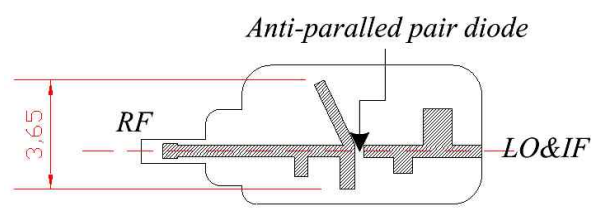

Fig. 8. Anti-parallel pair diode mixer module.

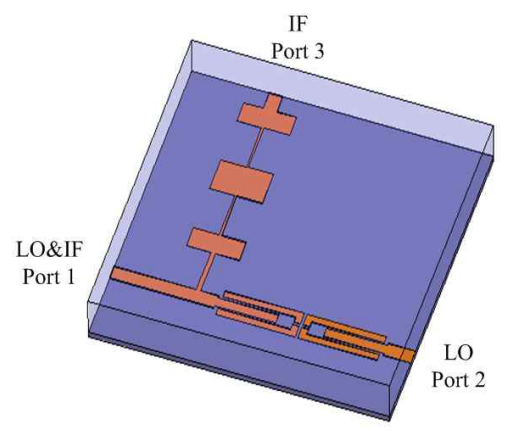

Fig. 9. The diplexer. 
output port is less than $-30 \mathrm{~dB}$.

The S-parameters of the diplexer are tested by vector network analyzer (Agilent E8563C). The two ports of VNA connect the two of 3 ports of the diplexer and the other port is matched by $50 \mathrm{ohm}$ load. The measured results denoted by the solid triangle are shown in Fig. 10. The measured insertion loss of bandpass filter at $23.15 \mathrm{GHz}$ is $1.5 \mathrm{~dB}$. The measured $3 \mathrm{~dB}$ cutoff frequency of the lowpass filter is $8 \mathrm{GHz}$. The isolation at $23.15 \mathrm{GHz}$ between LO input port and IF output port is less than $-35 \mathrm{~dB}$. The measurement results have good agreement with the simulation.

When LO frequency is $23.15 \mathrm{GHz}$ and LO power is $9.73 \mathrm{dBm}$, the simulation result is shown in Fig. 11. When RF frequency is $91 \mathrm{GHz}$, the best conversion loss is $15 \mathrm{~dB}$. When $\mathrm{RF}$ is between $87.6 \mathrm{GHz}$ and 99.4 $\mathrm{GHz}$, the conversion loss is less than $20 \mathrm{~dB}$, and the RF bandwidth is about $11 \mathrm{GHz}$.

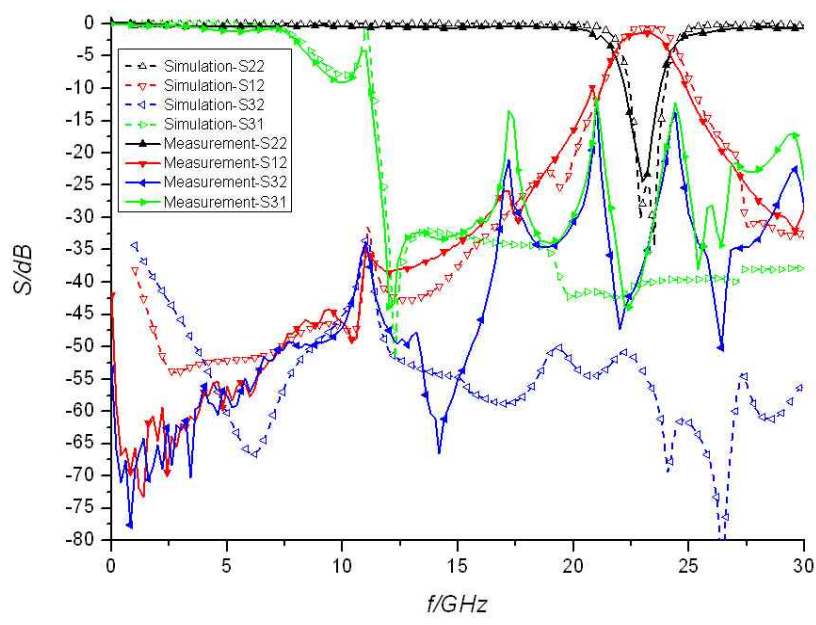

Fig. 10. The diplexer simulation and measurement results.

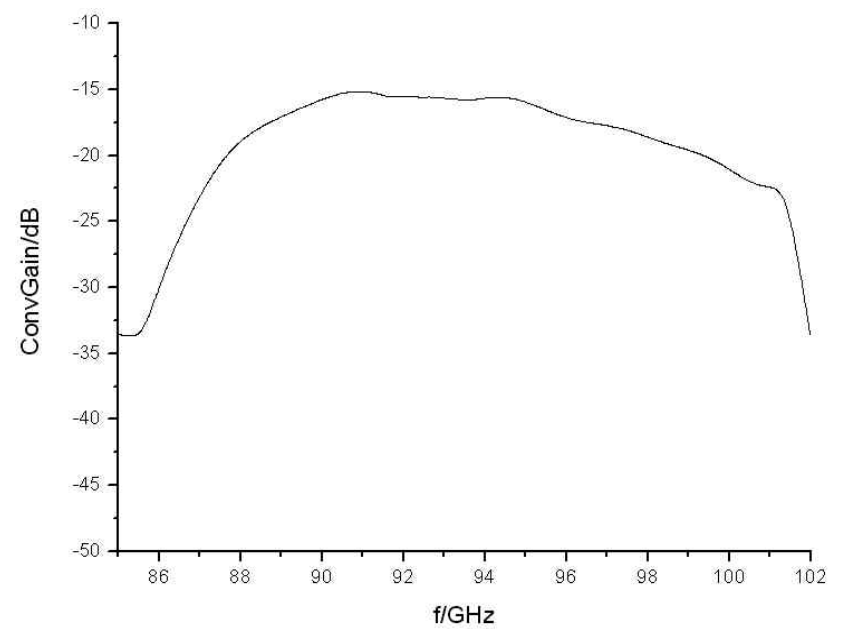

Fig. 11. Conversion loss simulation curve with the RF frequency.



Fig. 12. Test diagram.

\section{3-4 Measurements}

The test diagram of the novel fourth harmonic mixer is shown in Fig. 12. The RF source is a source module controlled by an Agilent signal generator. The LO source is a signal generator (Agilent E8257D). The IF signals is received by a spectrum analyzer (Agilent E4440A).

The photograph of the novel fourth harmonic mixer is shown in Fig. 13. The cavity size of the anti-parallel pair diode module is $20.5 \times 10.1 \mathrm{~mm}$; the circuit size of the anti-parallel pair diode module is $10.84 \times 3.65 \mathrm{~mm}$. The cavity size of the diplexer is $27.1 \times 26 \mathrm{~mm}$. The circuit size of the diplexer is $22.41 \times 16.85 \mathrm{~mm}$. The outside diameter of the coaxial cable is only $2.2 \mathrm{~mm}$, which connects the anti-parallel pair diode module with the diplexer. The anti-parallel pair diode module includes a waveguide/microstrip transmission.

The anti-parallel pair diode module is a component with two ports, so many novel fourth harmonic mixers are easy to be placed on the focus plane. This way can solve the puzzle of the traditional 3-ports mixer in the imaging system.

With LO frequency of $23.20 \mathrm{GHz}$ and LO power of $16 \mathrm{dBm}$, the measurement result is shown in Fig. 14.

The best conversion loss is $16.3 \mathrm{~dB}$ at $\mathrm{RF}$ signal of 91.3 GHz. The conversion loss is less than $20 \mathrm{~dB}$ with $\mathrm{RF}$ frequency of $88 \sim 94.4 \mathrm{GHz}$, so the RF bandwidth is about $6 \mathrm{GHz}$. The conversion loss measured curve is different from simulation result especially in the upper frequency band above $93 \mathrm{GHz}$ because many experimental factors are difficult to be built accurate model, for example, the diode spice model parameters, adhesive impedance of the diode assembly and fabrication errors, etc.

A line array consists of several novel fourth harmonic



Fig. 13. The photograph of the novel fourth harmonic mixer. 


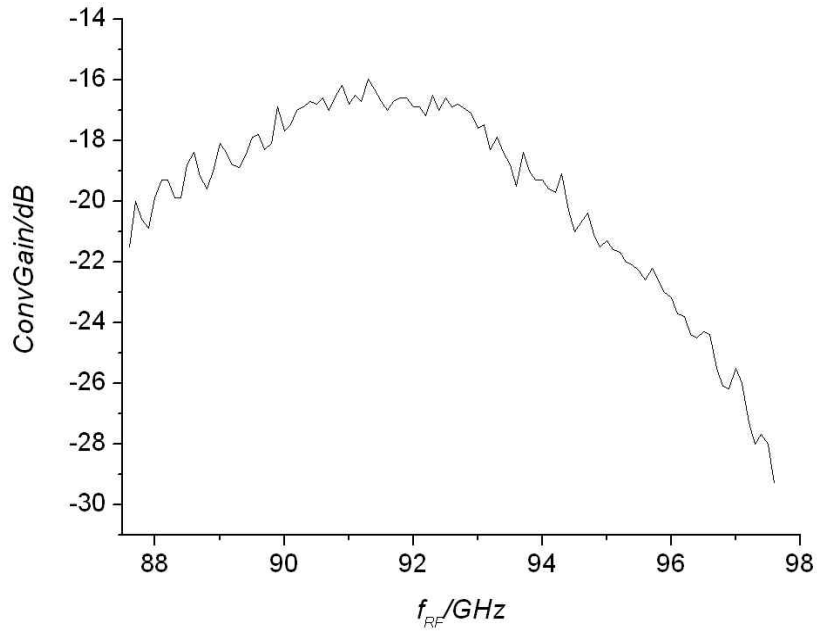

Fig. 14. The conversion loss measured curve with the RF frequency.

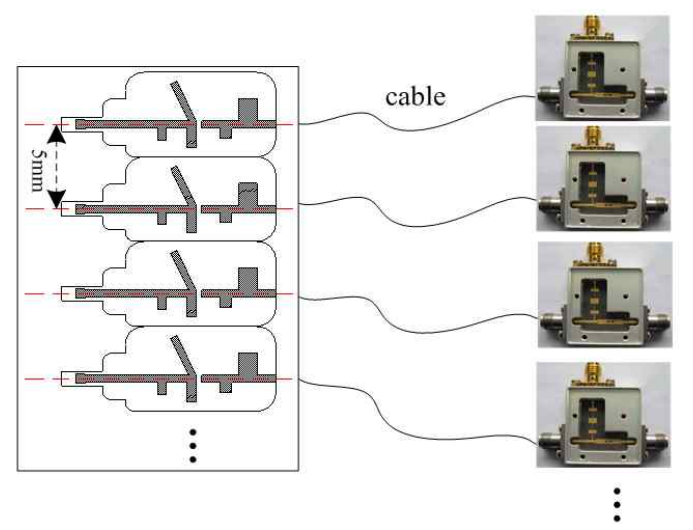

Fig. 15. Fourth harmonic mixer line array sketch.

mixers, as shown in Fig. 15. Similarly, a plane array consists of several line arrays. The fourth harmonic mixers array can be used in the imaging system.

\section{Conclusion}

The waveguide/microstrip transition, lowpass filter and bandpass filter are simulated and S-parameters are exported by HFSS. The open-circuited stubs or short-circuited stubs are simulated and S-parameters are exported by CST. All S-parameters and the diode spice model parameters are imported into ADS and the whole circuit system is simulated by ADS. In this paper, two kinds of fourth harmonic mixers are designed and optimized by using ADS, HFSS and CST. The traditional 3-ports fourth harmonic mixer is used in mechanical scanning equipment in the imaging system. The novel fourth harmonic mixer is more suitable for imaging system.

This work is supported by NSFC under grant 60921063 and innovation fund of state key Lab of MMW.

\section{References}

[1] Larry Yujiri, Merit Shoucri, and Philip Moffa, "Passive millimeter-wave imaging," IEEE Microwave, pp. 39-50, Sep. 2003.

[2] David M. Sheen, Douglas L. McMakin, and Thomas E. Hall, "Three-dimensional millimeter-wave imaging for concealed weapon detection," IEEE Transactions on Microwave Theory and Techniques, vol. 49, no. 9, Sep. 2001.

[3] Marvin Cohn, James E. Degenford, and Burton A. Newman, "Harmonic mixing with an anti-parallel diode pair," IEEE Transactions, vol. 23, pp. 171172, 1975.

[4] Yanfeng Xu, "A low conversion loss millimeter wave fourth subharmonic mixer," 2008 International Conference on Microwave and Millimeter Wave Technology Proceedings, ICMMT 2008, vol. 2, pp. 843-845, Nanjing, Apr. 2008.

[5] Zheng Lou, Sheng-Cai Shi, "A W-band eighth-order Schottky harmonic mixer," Microwave Conference, APMC 2009. Asia Pacific, pp. 610-613, Dec. 2009.

[6] D. Schneiderbanger, C. Kneuer, M. Sterns etc., "A 75-110 GHz seventh-harmonic balanced diode mixer in a novel circuit configuration," Wireless Technology, 2008. EuWiT 2008. European Conference, pp. 158-161, Oct. 2008.

[7] Sanjay Raman, Frank Rucky, and Gabriel M. Rebeiz, "A high-performance W-band uniplanar subharmonic mixer," IEEE Transactions on Microwave Theory and Techniques, vol. 45, no. 6, pp. 955-962, Jun. 1997.

[8] Minmin He, Wenbin Dou, "Microstrip probe/waveguide transition at $\mathrm{W}$ band," Microwave and Millimeterwave Symposium of China, vol. 87, pp. 353- 356, 2009.

[9] Meng-xia $\mathrm{Yu}$, Jun $\mathrm{Xu}$, and jian-xin Chen, "A novel millimeterwave microstrip bandpass filte," Journal of Microwave, vol. 22, no. 3, pp. 45-47, Jun. 2006. 


\section{Bo Xiang}

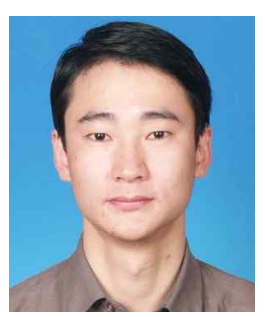

was born in Sichuan, China, in 1979. He received the B.S. degree from Chongqing University of Telecommunication and Posts, Chongqing, China, in 2002, and the M.S. degree from Southeast University, Nanjing, China, in 2010. Now he is Ph.D. student of the State Key Lab of Millimeter Waves, Southeast University. His current research includes millimeter circuit, power combiner and millimeter wave focal imaging.

\section{Wenbin Dou}

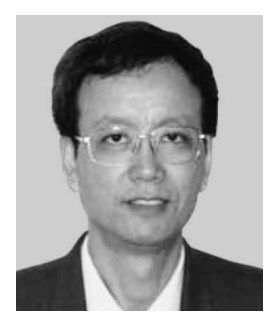

was graduated from University of Science and Technology of China, Hefei, in 1978. He received Master and Ph.D. degree from University of Electronic Science and Technology of China, Chengdu, in 1983 and 1987, both in Electronics and Communications. From 1987 to 1989 , he worked in Southeast University as postdoctoral fellow. Since 1989, he is with the Department of Radio Engineering, Southeast University. In 1994, he was promoted to professor. $\mathrm{He}$ is vice director of State Key Laboratory of Millimeter Waves. His research interesting include ferrite devices, millimeter wave quasi-optics, millimeter wave focal imaging, antennas and scattering, millimeter wave binary optics and so on. He had completed many projects on millimeter waves form State Ministries and Foundation and now is in charge of some key projects. He has published over 100 technique paper in Journals. He is author and co-author of two books. One is Millimeter wave ferrite device: theory and techniques(in Chinese) that is published in 1996. Another is Millimeter wave quasi optical theory and techniques (in Chinese) that is published in 2000 and re-published in 2006. He received many awards from State Ministry, Foundation. He is a member of State Ministry Expert Committee. He is also a member of editor committee of PIER and invited reviewer for the many magazines on Microwave and Optics in USA and China. He is a senior member of Chinese of CIE and member of IEEE and OSA.

\section{Minmin $\mathrm{He}$}

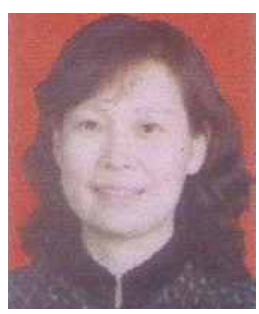

was graduated from Nanjing University of Aeronautics and Astronautics, Nanjing, in 1980. She worked in Nanjing Electronic Device Institute after graduation. Since 2000 she has been working in the State Key Laboratory of Millimeter Waves. Her research interests include device and circuit of microwaves and millimeter waves.

\section{Zongxin Wang}

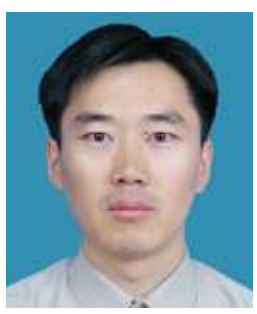

was born in Yantai, Shandong province, China, in 1970. He received the Doctor degree of Science (Tech.) in the Southeast University at 2005, China. His research interests include millimeter-wave imaging technology and electrically scanned antennas. 